\title{
Ocular involvement and visual outcome of herpes zoster ophthalmicus: review of 45 patients from Tunisia, North Africa
}

\author{
Rim Kahloun, Sonia Attia, Bechir Jelliti, Ahmed Zakaria Attia, Sana Khochtali, Salim Ben Yahia, Sonia Zaouali \\ and Moncef Khairallah*
}

\begin{abstract}
Background: Ocular complications of herpes zoster ophthalmicus (HZO) may lead to substantial visual impairment. The purpose of this study was to characterize and analyze ocular involvement and visual outcome of $\mathrm{HZO}$ in patients from Tunisia, North Africa. This study is a retrospective chart review of 51 eyes of 45 patients with HZO.

Results: Mean age was 44.5 years. Thirty patients (66.7\%) were aged over 50 years. Twenty-four patients (53.3\%) were male and 21 patients were female (46.7\%). There was no statistically significant difference in gender distribution. Initial mean best corrected visual acuity (BCVA) was 20/50. Ocular manifestations included adnexal involvement (58.8\%), keratitis (31.4\%), keratouveitis (31.4\%), isolated anterior uveitis (AU) (29.4\%), intraocular pressure elevation (23.5\%), oculomotor nerve palsy (5.8\%), and optic neuritis (1.9\%). Isolated $\mathrm{AU}(p<0.001)$, isolated keratitis $(p=0.001)$, and intraocular pressure elevation ( $p=0.013$ ) were more likely to be concomitant to HZO active skin disease, while keratouveitis occurred more likely more than 1 month after $\mathrm{HZO}$ eruption $(p<0.001)$. AU and keratouveitis were more likely to be associated with age $\geq 50$ years ( $p=0.001$ and $p=0.02$, respectively). Ocular complications included neurotrophic keratopathy (1.9\%), corneal opacity (5.9\%), secondary glaucoma (7.8\%), optic atrophy (1.9\%), and postherpetic neuralgia (13.3\%). Mean follow-up was 12 months. Mean final BCVA was 20/32; it was $\geq 20 / 40$ in $78.4 \%$ of the eyes.

Conclusions: Our study provided epidemiologic and clinical data of $\mathrm{HZO}$ in a Tunisian population. AU and keratitis were the most common ocular complications. Neurotrophic keratopathy was scarce. The overall visual outcome is good, with about three quarters of the treated patients maintaining VA of 20/40 or better.
\end{abstract}

Keywords: Herpes zoster ophthalmicus; Varicella zoster virus; Uveitis; Keratouveitis; Keratitis; Visual outcome; Epidemiology

\section{Background}

Herpes zoster ophthalmicus (HZO) is a reactivation of the varicella zoster virus involving the ophthalmic division of cranial nerve V. Herpes zoster affects $20 \%$ to $30 \%$ of the population at some point in their lifetime, and approximately $10 \%$ to $20 \%$ of these individuals will have HZO [1]. Ocular involvement occurs in approximately $50 \%$ of HZO patients in the absence of prompt preventive antiviral therapy [1]. HZO is often associated with a chronic course characterized by significant ocular morbidity and may lead to significant visual impairment in the absence of prompt and adequate treatment. Data on HZO from North African region are lacking.

* Correspondence: moncef.khairallah@rns.tn

Department of Ophthalmology, Fattouma Bourguiba University Hospital, Faculty of Medicine, University of Monastir, 5019 Monastir, Tunisia
The purpose of the present study was to characterize and analyze ocular involvement and visual outcome of HZO in patients from Tunisia, North Africa.

\section{Methods}

The charts of 45 consecutive patients (51 eyes) with HZO managed at the Department of Ophthalmology at Fattouma Bourguiba University Hospital of Monastir, Tunisia, from 1 January 2000 to 31 January 2012 were reviewed. The diagnosis of acute $\mathrm{HZO}$ was based on the presence of a primary vesiculomacular and dysesthetic skin rash within the ophthalmic dermatome. All patients were referred to our department from emergency department or by ophthalmologists. Patients were questioned concerning the history of the present illness, compromised immune 
status, medication use including immunosuppressive drug and topical or systemic antiviral therapy at presentation, renal failure, moderate or severe hypertension, diabetes mellitus, and previous relevant eye diseases such as chronic uveitis, keratitis, cataract, and glaucoma. All patients underwent detailed ophthalmic examination including adnexae examination, ocular motility, Snellen best corrected visual acuity (BCVA), slit-lamp examination, and fluorescein test. Adnexal involvement, including lid edema, blepharoconjunctivitis with macular rash involving the eyelids, conjunctival injection, vesicles, and petechial hemorrhages, was sought. Corneal sensitivity testing by comparison of a light touch with a cotton wool strand against the normal fellow eye was performed and recorded as normal, reduced, or absent. Grading of intraocular inflammation using standardization of uveitis nomenclature (SUN) working group criteria [2], tonometry, and dilated fundus examination with noncontact and contact lenses was also performed for all patients.

Ocular manifestations were defined as any ocular disease secondary to HZO. Intraocular pressure (IOP) elevation was defined as IOP $\geq 22 \mathrm{mmHg}$. The type of uveitis, onset, duration, and course was classified according to the SUN working group criteria [2]. Keratitis was divided into epithelial keratitis and stromal keratitis. When keratitis was associated to uveitis, we used the term keratouveitis.

Routine laboratory work-up including a complete blood count, erythrocyte sedimentation rate, C-reactive protein tests, and serum biochemical analysis was performed for all patients. Patients with bilateral involvement or aged less than 50 years underwent dermatologic and infectious diseases specialized examinations as well as serological examinations, including HIV antibodies and syphilis serology.

The presence or absence of postherpetic neuralgia (PHN) was also recorded. PHN was defined in our study as any symptom of pain or the use of pain medication for ocular symptoms documented in the medical record at least 3 months after onset of $\mathrm{HZO}$ [3].

Patients were divided into two groups: group I including patients with no ocular complications related to $\mathrm{HZO}$ and group II including patients with ocular complications related to $\mathrm{HZO}$. Group II was also divided into two subgroups: group $\mathrm{II}_{1}$ including patients with ocular complications concomitant to $\mathrm{HZO}$ and group $\mathrm{II}_{2}$ including patients with ocular complications occurring at least 1 month after $\mathrm{HZO}$ (Table 1).

All patients were treated with intravenous acyclovir $10 \mathrm{mg} / \mathrm{kg} 3$ times daily or oral valacyclovir $3 \mathrm{~g} /$ day for 7 to 10 days. Topical corticosteroids, cycloplegics, topical antibiotics, topical beta-blockers, oral carbonic anhydrase inhibitors, and analgesics were prescribed when indicated. Patients with anterior uveitis (AU) received antiviral therapy for 8 to 14 weeks along with topical corticosteroid therapy tapering. The mean follow-up was 12 months (range 9 to 51 ).

Data entry and statistical analyses were performed using Windows-based SPSS statistical software. Proportions were compared between groups using the chi-square or Fisher's exact test, as appropriate. Association between variables was considered statistically significant for a confidence level of $95 \%(p \leq 0.05)$.

\section{Results}

Demographic data of our patients are given in Table 2. Mean age was 44.5 years (range 12 to 77 ). Thirty patients (66.7\%) were aged over 50 years. There was no statistically significance in gender distribution in patients aged less or over 50 year old $(p=0.214)$ or in mean age between genders $(p=0.615)$. HZO was unilateral in 39 patients (86.7\%). HZO affected the right eye in 21 patients (46.6\%), the left eye in 18 patients (40\%), and both eyes in 6 patients (13.3\%). Associated systemic diseases included diabetes mellitus (9 patients; $20 \%$ ), systemic hypertension (8 patients; 17.8\%), and dyslipidemia (2 patients; $4.4 \%$ ). Used medication included long-term corticosteroid therapy for back pain (1 patient; $2.2 \%$ ) and immunosuppressive therapy for leukemia (1 patient; $2.2 \%$ ) or for renal transplantation (1 patient; $2.2 \%)$. Serology testing revealed HIV infection in 2 patients (4.4\%).

Of 6 patients with bilateral simultaneous HZO, 2 had diabetes, 2 were under immunosuppressive therapy and 1 had HIV infection, and the past medical history was unremarkable in the remaining patient.

Ocular symptoms included a red and painful eye in 42 patients $(93.3 \%)$, blurred vision in 44 eyes $(86.2 \%)$, and diplopia in 3 patients $(6.7 \%)$. Initial BCVA ranged from $20 / 200$ to $20 / 20$ (mean 20/50). It was $<20 / 200$ in 7 eyes (13.7\%) and $\geq 20 / 40$ in 15 eyes (29.4\%). Group I included 4 eyes $(7.8 \%)$ of 4 patients and group II 47 eyes $(92.2 \%)$ of 41 patients, divided into group $\mathrm{II}_{1}$ (32 eyes; $62.7 \%$ ) and group $\mathrm{II}_{2}$ (15 eyes; 29.5\%) (Table 1 ).

Ocular manifestations included adnexal involvement (30 eyes; 58.8\%), AU (31 eyes; 60.7\%), keratitis with corneal hypoesthesia (16 eyes; $31.4 \%$ ), oculomotor nerve palsy (3 eyes; $5.8 \%$ ), and optic neuritis with optic disc edema (1 eye; $1.9 \%)$. No case of retinal involvement was recorded in our patients.

Adnexal involvement included lid edema (30 eyes; $58.8 \%$ ), subconjunctival hemorrhage (23 eyes; $45.1 \%$ ), and vesicular conjunctivitis (8 eyes; $25.8 \%$ ).

Corneal hypoesthesia was recorded in 16 eyes (31.4\%). Corneal involvement included stromal keratitis in 8 eyes (15.6\%), epithelial keratitis in 5 eyes (9.8\%), and concomitant superficial and stromal keratitis in 3 eyes (5.8\%). Endotheliitis was not recorded in any affected eye.

Keratouveitis and isolated AU were observed respectively in 16 eyes (31.4\%) and in 15 eyes (29.4\%). AU was 
Table 1 Distribution of patients

\begin{tabular}{lcc}
\hline Groups & Number of eyes $\mathbf{N = 5 1}$ & $\%$ \\
\hline Group I: patients with no ocular complications related to HZO & 4 & 7.8 \\
Group II: patients with ocular complications related to HZO & 47 & 92.2 \\
Group II: patients with ocular complications concomitant to HZO & 32 & 62.7 \\
Group II2: patients with ocular complications occurring at least 1 month after HZO & 15 & 29.5 \\
\hline
\end{tabular}

$\mathrm{HZO}$, herpes zoster ophthalmicus.

acute in 26 eyes $(83.8 \%)$, recurrent in 2 eyes (6.4\%), and chronic in 3 eyes $(6.9 \%)$. Granulomatous keratic precipitates were found in 18 eyes $(58.1 \%)$. Focal iris atrophy was recorded in 8 eyes $(25.8 \%)$, Koeppe's nodules in 2 eyes $(6.5 \%)$, pupillary distortion in 2 eyes (6.5\%), and posterior synechiae in 5 eyes (16.1\%). IOP elevation was noted in 12 eyes of 31 eyes with AU (23.5\%) (Table 3).

Oculomotor nerve palsy included sixth cranial nerve in 2 eyes (3.9\%) and third cranial nerve in 1 eye (1.9\%).

All patients received antiviral therapy 2 to 10 days (average of 4 days) after the onset of ocular symptoms. Keratitis gradually improved in all patients. There was neurotrophic keratopathy in 1 eye (1.9\%) of a patient aged over 50 years and corneal opacity in 3 eyes (5.9\%). IOP elevation was controlled with medical therapy in 8 $(15.7 \%)$ of the 12 eyes with IOP elevation. Four eyes (7.8\%) developed secondary glaucoma with optic disc cupping and visual field defects.

Oculomotor nerve palsy gradually resolved within few months in all 3 eyes initially involved. The case of optic neuritis developed optic disc atrophy with deep VA loss.

Table 2 Demographic characteristics and distribution of patients with herpes zoster ophthalmicus

\begin{tabular}{|c|c|c|}
\hline & Number of patients $N=45$ & $\%$ \\
\hline \multicolumn{3}{|l|}{ Age } \\
\hline Mean age & 44.5 years (range 12 to 77 ) & \\
\hline \multicolumn{3}{|l|}{ Intervals } \\
\hline$<50$ & 15 & 33.3 \\
\hline 50 to 70 & 24 & 53.3 \\
\hline$\geq 70$ & 6 & 13.3 \\
\hline \multicolumn{3}{|l|}{ Gender } \\
\hline Male & 24 & 53.3 \\
\hline Female & 21 & 43.7 \\
\hline \multicolumn{3}{|l|}{ Laterality } \\
\hline Unilateral & 39 & 86.7 \\
\hline Bilateral & 6 & 13.3 \\
\hline \multicolumn{3}{|l|}{ Risk factors } \\
\hline Diabetes & 9 & 20 \\
\hline Immunosuppressive therapy & 2 & 4.4 \\
\hline Long-term corticosteroid therapy & 1 & 2.2 \\
\hline HIV infection & 2 & 4.4 \\
\hline
\end{tabular}

Mean final BCVA was 20/32 (range 20/400 to 20/20). It was $<20 / 200$ in 4 eyes $(7.8 \%)$ and $\geq 20 / 40$ in 40 eyes (78.4\%).

Our statistical analysis showed that patients from group $\mathrm{II}_{1}$ are more likely to have isolated $\mathrm{AU}(p<0.001)$, isolated keratitis $(p=0.001)$, and IOP elevation $(p=0.013)$ than patients with ocular complications occurring at least 1 month after HZO. However, keratouveitis occurred more likely in group $\mathrm{II}_{2}(p<0.001)$. AU and keratouveitis were more likely to be associated with age $\geq 50$ years $(p=0.001$ and $p=0.02$, respectively). There was no statistically significant relationship between ocular complications of $\mathrm{HZO}$ and gender (Table 4). PHN was recorded in 6 patients (13.3\%).

\section{Discussion}

The estimates of herpes zoster ranged between 320 and 410 per 100,000 person-years [4]. A recent study from the Pacific region estimates the overall incidence rate of

Table 3 Ocular complications associated with herpes zoster ophthalmicus

\begin{tabular}{lcl}
\hline & Number of eyes $\mathbf{N}=\mathbf{5 1}$ & \% \\
\hline Lid edema & 30 & 58.8 \\
Subconjunctival hemorrhage & 23 & 45.1 \\
Vesicular conjunctivitis & 8 & 15.7 \\
Corneal hypoesthesia & 16 & 31.4 \\
Keratitis & 16 & 31.4 \\
$\quad$ Epithelial keratitis & 5 & 9.8 \\
Stromal keratitis & 8 & 15.7 \\
$\quad$ Epithelial and stromal keratitis & 3 & 5.9 \\
Anterior uveitis only & 15 & 29.4 \\
Keratouveitis & 16 & 31.4 \\
Iris atrophy & 8 & 15.7 \\
Posterior synechiae & 5 & 9.8 \\
Pupillary distortion & 2 & 3.9 \\
IOP elevation & 12 & 23.5 \\
Glaucoma & 4 & 7.8 \\
Neurotrophic keratopathy & 1 & 1.9 \\
Oculomotor nerve palsy & 3 & 5.9 \\
Optic neuritis & 1 & 1.9 \\
\hline IOP, intraocular pressure. & &
\end{tabular}


Table 4 Correlations between ocular complications in herpes zoster ophthalmicus groups, age, and gender

\begin{tabular}{|c|c|c|c|c|c|c|c|c|c|c|}
\hline \multirow[t]{2}{*}{ Ocular involvement } & \multicolumn{3}{|l|}{ Group } & \multicolumn{3}{|c|}{ Age (years) } & \multicolumn{3}{|l|}{ Gender } & \multirow[t]{2}{*}{ Total } \\
\hline & $I I_{1} \%$ & $\mathrm{II}_{2} \%$ & $p$ value & $\geq 50 \%$ & $<50 \%$ & $p$ value & Female \% & Male \% & $p$ value & \\
\hline Anterior uveitis & 38.3 & 27.66 & 0.27 & 45.10 & 15.69 & $0.001^{*}$ & 33.33 & 27.45 & 0.51 & 31 \\
\hline Isolated anterior uveitis & 31.91 & 0 & $<0.001^{*}$ & 19.61 & 9.80 & 0.16 & 21.57 & 7.84 & 0.051 & 15 \\
\hline Keratouveitis & 6.38 & 27.66 & $<0.001^{*}$ & 23.53 & 7.84 & $0.02^{*}$ & 17.65 & 13.73 & 0.58 & 16 \\
\hline Isolated keratitis & 29.79 & 4.26 & $0.001^{*}$ & 15.69 & 15.69 & 1 & 11.76 & 19.61 & 0.27 & 16 \\
\hline IOP elevation & 21.28 & 4.26 & $0.013^{*}$ & 15.69 & 7.84 & 0.21 & 9.80 & 13.73 & 0.53 & 12 \\
\hline Oculomotor nerve palsy & 6.38 & 0 & 0.24 & 5.88 & 0 & 0.24 & 3.92 & 1.96 & 1 & 3 \\
\hline Iris atrophy & 6.38 & 10.64 & 0.71 & 13.73 & 1.96 & 0.07 & 11.76 & 3.92 & 0.26 & 8 \\
\hline
\end{tabular}

$\mathrm{IOP}$, intraocular pressure. *statistically significant $\mathrm{p}$ values.

HZO at 30.9 per 100,000 person-years, which is approximately $10 \%$ of all estimates of herpes zoster overall [5].

In our study, $66.7 \%$ of patients were aged over 50 years, and $53.3 \%$ of patients were aged between 50 and 70 years. Previous studies showed that the incidence of $\mathrm{HZO}$ increases significantly with age $[1,5]$. A recent case series reports that $\mathrm{HZO}$ affects similarly individuals aged younger than or older than 60 years, with the most common decade of onset between age 50 and 59 years [6]. In a recent study, the incidence rate for the subgroup of the population older than 65 years was approximately five times that of the rest of the population [5]. Increased age has been associated with a decrease in cell-mediated immunity, which is a crucial factor to avoid reactivation of the latent varicella zoster virus.

Ghaznawi et al. [6] showed that younger individuals were more likely to be healthy, with predisposing conditions such as immunosuppressive treatment, cancer, and HIV infection present in only $11.1 \%$ of patients who were younger at onset. In our study, immunosuppressive therapy and HIV infection were recorded in 3 (20\%) of 15 patients aged less than 50 years.

The results of our study, consistent with data from a previous report [5], show no significant difference in $\mathrm{HZO}$ incidence between men and women overall and between specific age groups. However, some other studies found significant differences between incidence rates for men and women within specific age groups, but there was limited evidence of a significant difference overall between incidence rates for men and women $[4,7]$.

In the current study, a high incidence of ocular involvement due to HZO (92.2\%) was found. This may be explained by a bias selection since, in our hospital, the majority of $\mathrm{HZO}$ cases without ocular complaints are usually referred to infectious disease specialists or dermatologists.

$\mathrm{AU}$, with or without associated keratitis, was the most common ocular manifestation of $\mathrm{HZO}$ in our series, occurring in $60.8 \%$ of eyes, followed by epithelial and/or stromal keratitis recorded in $31.4 \%$ of eyes. Similarly, in a study including 73 immunocompetent patients from the Netherlands during a period of 6 months follow-up, AU was reported in $43.8 \%$ of eyes at initial examination, followed by stromal keratitis in $30.1 \%$ of eyes [8]. In another study, AU was recorded in $46.6 \%$ of cases and keratitis in $76.2 \%$ of cases [9]. In a series of 18 younger than 40 years old patients with $\mathrm{HZO}, \mathrm{AU}$ was recorded in $50 \%$ of cases and stromal keratitis in $22 \%$ of cases [10]. In a recent study from the Pacific Islanders, a lower rate of $\mathrm{AU}$ was recorded (6\% of cases); however, keratitis was recorded in $28.3 \%$ of cases [5]. In the latter study, all patients, with or without ocular involvement, were included; this may explain the lower proportion of AU.

In the current study, isolated AU and isolated keratitis were more likely to be concomitant to HZO eruption ( $p<0.001$ and $p=0.001$, respectively); however, keratouveitis is more likely to occur at least 1 month after HZO $(p<0.001)$. A recent study shows the risk of AU increased significantly in the year following a diagnosis of HZO [11]. In addition, we found that both isolated $\mathrm{AU}$ and keratouveitis were more likely to occur in patients $\geq 50$ years old ( $p=0.001$ and $p=0.02$, respectively), and there was no statistically significant association between age and the occurrence of keratitis. In contrast, a recent study comparing the course of HZO in patients' younger vs. older than 60 years found that younger patients had more episodes of delayed pseudodendritiform keratitis and AU compared with older patients. However, prevalences of corneal perforation, corneal thinning, cataract formation, and glaucoma were similar between the two groups [6].

Despite the fact that about one third of our patients developed keratitis, only one patient aged over 50 years old developed neurotrophic keratopathy. This may be explained by the early antiviral therapy in our patients with an average of 4 days after the onset of HZO. While Ghaznawi et al. [6] found that neurotrophic keratopathy affected $19.6 \%$ of all patients and was more frequent in older patients. Similarly, Liesegang et al. [12] reported a $25 \%$ prevalence of neurotrophic keratitis. 
In our series, oculomotor nerve palsy was recorded in $5.8 \%$ of eyes with complete resolution during follow-up. The reported incidence of extraocular muscle palsies in $\mathrm{HZO}$ ranged between $7 \%$ and $31 \%[13,14]$, but many cases are asymptomatic and usually transient. The third oculomotor nerve is most commonly affected, followed by the abducens nerve and the trochlear nerve [15]. Ophthalmoplegia was also reported $[14,16]$.

In our study, optic neuritis was recorded in only one eye. It is very rare sequelae of $\mathrm{HZO}$, that may occur simultaneously to the acute vesicular rash or, more frequently, as a postherpetic complication, up to 10 weeks after disease onset $[17,18]$. Optic neuritis is characterized by low vision and central visual field defects, with an optic nerve that looks initially normal or edematous, and which progresses to optic atrophy leading to visual loss like in our patient. A good recovery of HZO optic neuritis with systemic acyclovir and steroid has been also reported [18].

The overall visual outcome was relatively good in our series, with $78.4 \%$ of eyes having a final BCVA $\geq 20 / 40$, and only $7.8 \%$ of affected eyes were with final BCVA $<20 /$ 200. Causes of low vision included optic disc atrophy related to optic neuritis, neurotrophic keratopathy, and corneal opacity. Zaal et al. [8] reported a final VA > 20/ 25 in $90.4 \%$ of cases at 6 months follow-up. As well, Gupta et al. [10] reported a final BCVA of 20/40 or better in $90 \%$ of immunocompetent subjects younger than 40 years old. In a series of ten healthy children with $\mathrm{HZO}$, final BCVA was $20 / 20$ in $80 \%$ of cases [19]. In a prospective study, $56.3 \%$ of patients had a VA of $6 / 6$ or better and visual loss was associated to increasing age, positive Hutchinson's sign, absent corneal sensation, corneal epithelial lesions, and uveitis [20].

In this study, a lower rate of PHN (13.3\%) was found than those of previous studies [5,9,21]. A higher incidence (17.8\%) was reported by Ghaznawi et al. [6] in an American series including 112 patients and was statistically significantly higher in the group aged more than 60 years old. As well, in a Pacific region study, PHN was recorded in $20.9 \%$ of patients [5], with $64.3 \%$ of patients older than 65 years. The main risk factor for PHN is advancing age; other risk factors include severity of acute zoster pain and rash, a painful prodrome, and ocular involvement $[1,22]$. It has been also shown that patients with keratitis, conjunctivitis, or uveitis had a higher risk of developing PHN compared with patients who did not have these ocular features [5].

Our study presents some limitations, related to its retrospective design, bias selection, and varying followup. We acknowledge that the use of final VA introduces a potential bias because the disease may be recurrent and continue to cause loss of VA beyond the time the outcome is reported. In addition to these limits, a major drawback is the potential bias selection.

\section{Conclusions}

Our study provided epidemiologic and clinical data of $\mathrm{HZO}$ in a Tunisian population. AU and keratitis were the most common ocular manifestations. Neurotrophic keratopathy was scarce.

The overall visual outcome is good, with about three quarters of the treated patients maintaining VA of 20/40 or better.

\section{Abbreviations}

AU: anterior uveitis; BCVA: best corrected visual acuity; HZO: herpes zoster ophthalmicus; IOP: intraocular pressure; PHN: postherpetic neuralgia; VA: visual acuity.

\section{Competing interests}

The authors declare that they have no competing interests.

\section{Authors' contributions}

RK, SA, BJ, and AZA have made substantial contributions to the conception and design, or acquisition of data, or analysis and interpretation of data. RK, SA, SZ, SZ, SBY, and MK have been involved in drafting the manuscript or revising it critically for important intellectual content. MK has given the final approval of the version to be published. RK and MK agree to be accountable for all aspects of the work in ensuring that the questions related to the accuracy or integrity of any part of the work are appropriately investigated and resolved. All authors read and approved the final manuscript.

\section{Acknowledgements}

This work has been supported by the Ministry of Higher Education and Research of Tunisia.

Received: 19 July 2014 Accepted: 8 September 2014

Published online: 17 September 2014

\section{References}

1. Liesegang TJ (2008) Herpes zoster ophthalmicus natural history, risk factors, clinical presentation, and morbidity. Ophthalmology 115(2 Suppl):S3-12

2. Jabs DA, Nussenblatt RB, Rosenbaum JT; Standardization of Uveitis Nomenclature (SUN) Working Group (2005) Standardization of uveitis nomenclature for reporting clinical data. Results of the first international workshop. Am J Ophthalmol 140:509-516

3. Dworkin RH, Schmader RE (2003) Treatment and prevention of post herpetic neuralgia. Clin Infect Dis 36:877-882

4. Cebrián-Cuenca AM, Díez-Domingo J, San-Martín-Rodríguez M, Puig-Barberá J, Navarro-Pérez J, Herpes Zoster Research Group of the Valencian Community (2011) Epidemiology and cost of herpes zoster and postherpetic neuralgia among patients treated in primary care centres in the Valencian Community of Spain. BMC Infect Dis 1(11):302

5. Borkar DS, Tham VM, Esterberg E, Ray KJ, Vinoya AC, Parker JV, Uchida A, Acharya NR (2013) Incidence of herpes zoster ophthalmicus: results from the Pacific ocular inflammation study. Ophthalmology 120:451-456

6. Ghaznawi N, Virdi A, Dayan A, Hammersmith KM, Rapuano CJ, Laibson PR, Cohen EJ (2011) Herpes zoster ophthalmicus: comparison of disease in patients 60 years and older versus younger than 60 years. Ophthalmology 118:2242-2250

7. Mullooly JP, Riedlinger K, Chun C, Weinmann S, Houston H (2005) Incidence of herpes zoster, 1997-2002. Epidemiol Infect 133:245-253

8. Zaal MJ, Völker-Dieben HJ, D'Amaro J (2003) Visual prognosis in immunocompetent patients with herpes zoster ophthalmicus. Acta Ophthalmol Scand 81:216-220

9. Yawn BP, Wollan PC, St Sauver JL, Butterfield LC (2013) Herpes zoster eye complications: rates and trends. Mayo Clin Proc 88:562-570

10. Gupta N, Sachdev R, Sinha R, Titiyal JS, Tandon R (2011) Herpes zoster ophthalmicus: disease spectrum in young adults. Middle East Afr J Ophthalmol 18:178-182

11. Wang TJ, Hu CC, Lin HC (2012) Increased risk of anterior uveitis following herpes zoster: a nationwide population-based study. Arch Ophthalmol 130:451-455 
12. Liesegang TJ (1985) Corneal complications from herpes zoster ophthalmicus. Ophthalmology 92:316-324

13. Park KC, Yoon SS, Yoon JE, Rhee HY (2011) A case of herpes zoster ophthalmicus with isolated trochlear nerve involvement. J Clin Neurol 7:47-49

14. Chhabra MS, Golnik KC (2014) Recovery of ocular motor cranial nerve palsy after herpes zoster ophthalmicus. J Neuroophthalmol 34:20-22

15. Tsuda H, Ito T, Yoshioka M, Ishihara N, Sekine Y (2007) Isolated trochlear nerve palsy in herpes zoster ophthalmicus. Intern Med 46:535-536

16. Hakim W, Sherman R, Rezk T, Pannu K (2012) An acute case of herpes zoster ophthalmicus with ophthalmoplegia. Case Rep Ophthalmol Med 2012:953910

17. De Mello VB, Foureaux EC, Porto FB (2011) Herpes zoster optic neuritis. Int Ophthalmol 31:233-236

18. Wang AG, Liu JH, Hsu WM, Lee AF, Yen MY (2000) Optic neuritis in herpes zoster ophthalmicus. Jpn J Ophthalmol 44:550-554

19. De Freitas D, Martins EN, Adan C, Alvarenga LS, Pavan-Langston D (2006) Herpes zoster ophthalmicus in otherwise healthy children. Am J Ophthalmol 142:393-399

20. Nithyanandam S, Stephen J, Joseph M, Dabir S (2010) Factors affecting visual outcome in herpes zoster ophthalmicus: a prospective study. Clin Experiment Ophthalmol 38:845-850

21. Puri LR, Shrestha GB, Shah DN, Chaudhary M, Thakur A (2011) Ocular manifestations in herpes zoster ophthalmicus. Nepal J Ophthalmol 3:165-171

22. Opstelten W, Mauritz JW, de Wit NJ, van Wijck AJ, Stalman WA, van Essen GA (2002) Herpes zoster and postherpetic neuralgia: incidence and risk indicators using a general practice research database. Fam Pract 19:471-475

doi:10.1186/s12348-014-0025-9

Cite this article as: Kahloun et al:: Ocular involvement and visual outcome of herpes zoster ophthalmicus: review of 45 patients from Tunisia, North Africa. Journal of Ophthalmic Inflammation and Infection 2014 4:25.

\section{Submit your manuscript to a SpringerOpen ${ }^{\circ}$ journal and benefit from:}

- Convenient online submission

- Rigorous peer review

- Immediate publication on acceptance

- Open access: articles freely available online

- High visibility within the field

- Retaining the copyright to your article

Submit your next manuscript at $\gg$ springeropen.com 